\title{
Conjectural variations and evolutionary stability in finite populations
}

\author{
Wieland Müller • Hans-Theo Normann
}

Published online: 2 December 2006

(C) Springer-Verlag 2006

\begin{abstract}
Recently it has been shown that consistent conjectures are evolutionarily stable. In this note we show that this finding depends on the use of the infinite population ESS (Maynard-Smith, Evolution and the theory of games, Cambridge University Press, Cambridge, UK, 1982). When applying the finitepopulation ESS (Schaffer, J Theor Biol 132:469-478, 1988) we show that the conjectures surviving in the long run are not consistent.
\end{abstract}

Keywords Consistent conjectures • Duopoly • Evolutionary stability • Finite population $\cdot$ Indirect evolutionary approach

\section{JEL Classification D43}

\section{Introduction}

Dixon and Somma (2003) as well as Müller and Normann (2005) have recently shown that consistent conjectures (Bresnahan 1981) are evolutionarily stable. Possajennikov (2004) extends these results to general two-player games. In this note, we show that this finding depends on assuming an infinite population and, accordingly, the use of the infinite-population ESS (Maynard-Smith 1982). Here, we assume a finite population of size $N<\infty$ and apply the finitepopulation ESS as introduced by Schaffer (1988). By virtue of the properties

W. Müller $(\varangle)$

Department of Economics, Tilburg University, Postbus 90153,

5000 LE Tilburg, The Netherlands

e-mail: w.mueller@uvt.nl

\section{H.-T. Normann}

Department of Economics, Royal Holloway, Egham, Surrey TW20 0EX, UK

e-mail: hans.normann@rhul.ac.uk 
of this static finite-population ESS, we show that the conjectures surviving in the long run are not consistent. Only when the population size $N$ approaches infinity do the results provided here approach those reported in Müller and Normann (2005). In this sense, the results described in this paper generalize the ones reported in the earlier paper. They suggest that the evolutionary support of consistent conjectures is weaker in the more realistic setting of a finite population.

More generally, we show that the market outcome in a finite population will be more competitive than in an infinite population. In particular, when $N \rightarrow 2$, the market result approaches the perfectly competitive market outcome. This relates our results to several papers in the literature. Vega-Redondo (1997) considers an imitation dynamic in a Cournot market with a finite number of players who, as time proceeds, imitate the behavior of the player who had the highest profit in the previous period. This dynamic is shown to converge to the competitive outcome. Furthermore, Güth and Huck (1997) consider an infinite population of sellers who are repeatedly matched in pairs to act in a symmetric heterogenous-goods market. The authors assume that, although true profit functions reflecting some particular market structure exist, sellers may not be aware of them. Instead, sellers may entertain some beliefs about the market structure which leads them to maximize so-called conjectural profit functions. Güth and Huck (1997) show that conjectural profit functions neglecting strategic interdependence are (neutrally) evolutionarily stable. More precisely, it turns out that each seller in the evolutionary equilibrium thinks he would act in a monopoly market and, accordingly, produces monopoly quantities with regard to his conjectural profit function. Surprisingly, these individual quantities give rise to the competitive outcome with regard to the true profit functions.

\section{Assumptions}

Consider two firms $i=1,2$ in a heterogeneous-goods market where firms choose quantities. The inverse demand functions are given by

$$
p_{i}\left(q_{i}, q_{j}\right)=a-q_{i}-\theta q_{j}, \quad i, j=1,2 ; i \neq j
$$

with $0<\theta \leq 1$. The symmetric cost functions are

$$
C\left(q_{i}\right)=c\left(q_{i}\right)^{2} / 2, i=1,2,
$$

with $c \geq 0$. Firm $i$ 's profit reads

$$
\pi_{i}\left(q_{i}, q_{j}\right)=\left(a-q_{i}-\theta q_{j}\right) q_{i}-\frac{c}{2}\left(q_{i}\right)^{2} .
$$


Our assumptions on demand and cost in the Cournot case are equal to Assumptions 1 and 2 in Bresnahan (1981), except that we assume that firms are symmetric. $^{1}$

\section{Quantity competition}

We briefly reiterate Bresnahan's (1981) definition of a consistent conjectures equilibrium (CCE) with Cournot competition. Let $\rho_{i}=\rho_{i}\left(q_{j}\right), i \neq j$, denote firm $i$ 's best response function. From our assumptions, we know that a unique and linear CCE exists (Bresnahan 1981, Theorem 1). We therefore restrict attention to linear conjectures such that $r_{i} \in[-1,1], i=1,2$, denotes firm $i$ 's conjectures about firm $j$ 's reaction to $q_{i}$. We impose the restriction $r_{i} \in[-1,1]$ to guarantee equilibrium quantities to be non-negative. The outcomes in a symmetric Cournot duopoly range from perfect competition to joint monopoly when the conjectural variation increases from -1 to 1 .

Definition 1 A consistent conjectures equilibrium is a pair of quantities, $\left(q_{1}^{*}, q_{2}^{*}\right)$, and of conjectures, $\left(r_{1}^{*}, r_{2}^{*}\right)$, such that

$$
q_{1}^{*}=\rho_{1}\left(q_{2}^{*}\right), q_{2}^{*}=\rho_{2}\left(q_{1}^{*}\right),
$$

and

$$
r_{1}^{*}=\frac{d \rho_{2}\left(q_{1}\right)}{d q_{1}}, r_{2}^{*}=\frac{d \rho_{1}\left(q_{2}\right)}{d q_{2}} .
$$

That is, firms' quantities have to constitute a Nash equilibrium (conditions (2)), and a firm's conjecture about the other firm's behavior has to be equal to the slope of the other firm's reaction function (conditions (3)).

In Müller and Normann (2005) we show that, for the above market, the symmetric consistent-conjectures equilibrium is given by

$$
q_{i}^{*}=\frac{2 a}{2(1+\theta)+c+A},(i=1,2), \quad r^{*}=\frac{-2-c+A}{2 \theta}
$$

where $A:=\sqrt{(2+c)^{2}-4 \theta^{2}}>0$.

Instead of imposing a consistency condition as in Definition 1, one can make conjectures subject to evolutionary selection. For this purpose, we first derive firms' outputs given their conjectures. Since conjectures determine profits, they also determine reproductive success and one can study the evolutionary selection of conjectures in a second step. The underlying assumption is that, if firms differ in evolutionary success, the individual characteristics of a more successful firm will spread within the population more quickly than

\footnotetext{
${ }^{1}$ With asymmetric demand and cost functions, the evolutionary analysis below is extremely messy and cumbersome. Bresnahan (1981) shows that the model may also allow for fixed costs which, from his Assumption 3, should not be too large.
} 
the characteristics of a less successful one. This leads to a dynamic process that determines the long-run distribution of individual characteristics within an economy.

Consider the two steps more formally. Refer to firm $i$ 's (constant) conjecture, $r_{i}$, as to firm $i$ 's type. Firm types may be completely arbitrary and types are known whenever two firms compete with each other. First, we derive firms' behavior given their types. We assume here that firm $i$ will choose its quantity $q_{i}$ in order to maximize profits, that is, we assume that players act rationally given their types. Within strategic games this implies that the chosen strategy profile is a Nash equilibrium, denoted by $\left(q_{i}^{*}\left(r_{i}, r_{j}\right), q_{j}^{*}\left(r_{i}, r_{j}\right)\right)$. In the second step of evolutionary analysis, the types (conjectures) are the strategies and the evolutionary success function, that is, firm profits

$$
\pi_{i}^{*}\left(r_{1}, r_{2}\right) \equiv \pi_{i}\left(q_{1}^{*}\left(r_{i}, r_{j}\right), q_{2}^{*}\left(r_{i}, r_{j}\right)\right)
$$

evaluated at equilibrium strategies, are the payoff functions. To find the types that survive in the long run, in Müller and Normann (2005) we apply the ESS concept as introduced for an infinite population by Maynard-Smith (1982) and show that the conjecture $r^{*}$ given in Eq. 4 is evolutionarily stable. For this reason, let us denote $r_{\text {infinite }}^{*}:=(-2-c+A) / 2 \theta$ with $A$ as defined above.

In what follows, we will apply the ESS concept for a finite population as defined by Schaffer (1988) for the case of a two-player game. ${ }^{2}$ For this purpose, consider a population of size $N \geq 2$. Each time, two out of the $N$ individuals might be called to play a duopoly game. The question is whether a single mutant with conjecture $r$ can invade the population if all other individuals adhere to the conjecture $r^{*}$. This idea is captured by Shaffer's ESS definition used in condition (6) in the following definition.

Definition 2 An equilibrium with evolutionarily stable conjectures in a finite population is a pair of quantities, $\left(q_{1}^{*}, q_{2}^{*}\right)$, and a conjecture, $r^{*}$, such that

$$
q_{1}^{*}=\rho_{1}\left(q_{2}^{*}\right), q_{2}^{*}=\rho_{2}\left(q_{1}^{*}\right),
$$

and

$$
\pi_{1}^{*}\left(r, r^{*}\right) \leq\left(\frac{N-2}{N-1}\right) \pi_{2}^{*}\left(r^{*}, r^{*}\right)+\frac{1}{N-1} \pi_{2}^{*}\left(r^{*}, r\right) \text { for all } r \neq r^{*}
$$

That is, an equilibrium with evolutionarily stable conjectures requires a Nash equilibrium in outputs given the types (5), and an evolutionarily stable preference type $r^{*}$ such that no $r$-mutant invading a "society" of $r^{*}$-players can spread (Eq. 6).

\footnotetext{
${ }^{2}$ For another application of this ESS concept see e.g. Hehenkamp et al. (2004). 
We now solve for an equilibrium of this kind. The system of first-order conditions $\partial \pi_{i}\left(q_{i}, q_{j}\right) / \partial q_{i}=a-\theta q_{j}-q_{i}\left(2+\theta r_{i}+c\right)=0(i=1,2)$ can be solved for equilibrium strategies

$$
q_{i}^{*}\left(r_{i}, r_{j}\right)=\frac{a\left(2+\theta\left(r_{j}-1\right)+c\right)}{4(1+c)+\theta(2+c)\left(r_{i}+r_{j}\right)+\theta^{2}\left(r_{i} r_{j}-1\right)+c^{2}} .
$$

Substituting $q_{i}^{*}\left(r_{i}, r_{j}\right)$ and $q_{j}^{*}\left(r_{i}, r_{j}\right)$ in $\pi_{i}($.$) yields the evolutionary success$ $\pi_{i}^{*}\left(r_{i}, r_{j}\right)$ of type $r_{i}$, given that the opponent exhibits type $r_{j} \cdot{ }^{3}$

$$
\begin{aligned}
\pi_{i}^{*}\left(r_{i}, r_{j}\right) & =\pi_{i}\left(q_{i}^{*}\left(r_{i}, r_{j}\right), q_{j}^{*}\left(r_{i}, r_{j}\right)\right) \\
& =\frac{\left(q_{i}^{*}\right)^{2}}{2}\left(c+2\left(1+\theta r_{i}\right)\right) .
\end{aligned}
$$

Following Schaffer (1988), an ESS solves the maximization problem

$$
\max _{r}\left(\pi_{1}^{*}\left(r, r^{*}\right)-\left(1-\frac{1}{N-1}\right) \pi_{2}^{*}\left(r^{*}, r^{*}\right)-\frac{1}{N-1} \pi_{2}^{*}\left(r^{*}, r\right)\right) .
$$

Since $\pi_{2}^{*}\left(r^{*}, r^{*}\right)$ is a constant, this problem reduces to

$$
\max _{r}\left(\pi_{1}^{*}\left(r, r^{*}\right)-\frac{1}{N-1} \pi_{2}^{*}\left(r^{*}, r\right)\right) .
$$

Solving the first-order condition and using symmetry $\left(r=r^{*}\right)$ yields the following

Proposition 1 The unique evolutionarily stable conjecture of the quantity game is

$$
r_{\text {finite }}^{*}=\frac{-(2 N-c+N c+2 \theta-2)+B}{2 \theta(N-1)}
$$

where $B=\sqrt{(c+2)^{2}+N(c+2 \theta+2)(c-2 \theta+2)(N-2)}$.

(Another candidate can be excluded as it violates the assumptions set out in Section 2.) A few observations are in order.

- First, the conjecture in Proposition 1 does not coincide with the one given in Eq. 4. The proposition says that the evolutionarily stable conjecture in the finite-population case is not the consistent conjecture. Importantly, whereas in general $r_{\text {infinite }}^{*}>r_{\text {finite }}^{*}$, we find that $\lim _{N \rightarrow \infty} r_{\text {finite }}^{*}=r_{\text {infinite }}^{*}$.

\footnotetext{
${ }^{3}$ Note that the game with types $\left(\widehat{r}_{i}, \widehat{r}_{j}\right)$ does not have an equilibrium if $4(1+c)+$ $\theta(2+c)\left(\widehat{r}_{i}+\widehat{r}_{j}\right)+\theta^{2}\left(\widehat{r}_{i} \widehat{r}_{j}-1\right)+c^{2}=0$. For such $\left(\widehat{r}_{i}, \widehat{r}_{j}\right)$ we proceed as in Possajennikov (2000) by extending the fitness function by continuity in the first argument in the sense of $\pi_{i}^{*}\left(\widehat{r}_{i}, \widehat{r}_{j}\right)=$ $\lim _{r_{i} \rightarrow \widehat{r}_{i}} \lim _{r_{j} \rightarrow \widehat{r}_{j}} \pi_{i}^{*}\left(r_{i}, r_{j}\right)$. This limit does always exist on the extended real line $\mathbb{R} \cup\{ \pm \infty\}$ and, as a result, the function $\pi_{i}^{*}\left(r_{i}, r_{j}\right)$ is differentiable with respect to the first argument at $r_{j}=r_{i}$.
} 
- Second, we have $q_{i}^{*}\left(r_{\text {infinite }}^{*}, r_{\text {infinite }}^{*}\right)<q_{i}^{*}\left(r_{\text {finite }}^{*}, r_{\text {finite }}^{*}\right)$. This follows from $r_{\text {infinite }}^{*}>r_{\text {finite }}^{*}$ and $\partial q_{i}^{*}(r, r) / \partial r=-\theta a /(c+\theta+r \theta+2)^{2}<0$. This means that the market outcome in the case of a finite population will be more competitive than in the case of an infinite population.

- Third, we find $\lim _{N \rightarrow 2} r_{\text {finite }}^{*}=-1$. This implies that, for the border case of $N \rightarrow 2$, we approach the perfectly competitive market outcome. In this case, the evolutionarily stable conjecture is independent of underlying market parameters which is generally in contrast to the analysis with an infinitely large population in Müller and Normann (2005). However, for the special case of homogenous goods $(\theta=1)$ and constant marginal cost $(c=0)$, the consistent conjecture is -1 , and therefore finitely and infinitely large population analysis yield the same results again.

\section{Price competition}

In Müller and Normann (2005) we also consider price competition in addition to quantity competition. For this purpose, we invert the demand system (1) and impose $\theta<1$ strictly. We then show that the consistent-conjectures equilibrium for the price-setting market is given by

$$
\begin{aligned}
p_{i}^{*} & =\frac{a\left(2\left(1-\theta^{2}\right)+c(c+2+A)\right)}{(2+c+2 \theta)(1+c-\theta)+(c+1+\theta) A}, \\
r_{\text {infinite }}^{*} & =\frac{2\left(1-\theta^{2}\right)+c\left(1+\theta^{2}\right)+A\left(\theta^{2}-1\right)}{2 \theta\left(1-\theta^{2}+c\right)}
\end{aligned}
$$

where $A$ is defined as above for Cournot competition. Moreover, we show that the conjecture $r_{\text {infinite }}^{*}$ is evolutionarily stable when applying the ESS concept as introduced for an infinite population.

Applying the general definition of a finite-population ESS as defined in Eq. 6 for arbitrary $N \geq 2$ turns out to be extremely cumbersome. We therefore state here only the result for the extreme case of $N=2$. Proceeding as above one can proof the following

Proposition 2 The unique evolutionarily stable conjecture of the price game in the case of a population of size $N=2$ is $r_{\text {finite }}^{*}=-1$.

(The proof is given in the Appendix). We observe the following results. First, it turns out that the evolutionarily stable conjectures (in the finite population) in the Bertrand game are the same as in the Cournot game when $N=2$. In general, this is not true in the case of the infinite-population ESS. Second, this proposition says that the evolutionarily stable conjecture of the Bertrand game is inconsistent in the finite-population case (see Eq. 7). Third, again in contrast to Müller and Normann (2005), the evolutionarily stable conjecture is independent of underlying market parameters. 


\section{Discussion}

Why do the outcomes differ when we apply the finite-population ESS instead of the infinite-population ESS? The answer seems simple. In general, evolutionary forces direct individuals to maximize relative payoffs. In infinite populations, individuals cannot affect the average payoff of all other individuals. This implies that maximization of relative payoffs coincides with maximization of absolute payoffs for the infinite-population ESS.

However, for the finite-population ESS, this is not the case. Here individuals' concern for relative payoffs is not tantamount to a concern for absolute payoffs. In a population of only two players, we saw that players produce the perfectly competitive output. The reason is that each action directly affects the other player's profit, which forces players to maximize relative payoffs. With a large number of payers, the likelihood of affecting the payoff of other players becomes smaller and it becomes zero in the limit $N \rightarrow \infty$. In our model, the higher the quantity in the Cournot game, the higher a player's relative payoff in a finite population. In turn, the quantity in the Cournot game is the higher the lower is a player's conjecture. ${ }^{4}$ It follows that, the smaller the conjecture, the larger the output and, consequently, the larger a player's relative payoff.

Finally, we believe that the results derived here would hold in a more general framework that, in particular, would not make use of a parametrized demand system. However, we leave this to future research.

Acknowledgements We thank Werner Güth and an anonymous referee for very helpful comments. The first author acknowledges financial support from the Netherlands Organisation for Scientific Research (NWO) through a VIDI grant.

\section{Appendix}

\section{Proof of Proposition 2}

To analyze price competition, we need to invert the inverse demand functions given in Eq. 1 yielding

$$
q_{i}\left(p_{i}, p_{j}\right)=\frac{a(1-\theta)-p_{i}+\theta p_{j}}{1-\theta^{2}}, \quad i, j=1,2 ; i \neq j .
$$

and impose $\theta<1$ strictly. Firm $i$ 's profit reads

$$
\pi_{i}\left(p_{i}, p_{j}\right)=p_{i}\left(\frac{a(1-\theta)-p_{i}+\theta p_{j}}{1-\theta^{2}}\right)-\frac{c}{2}\left(\frac{a(1-\theta)-p_{i}+\theta p_{j}}{1-\theta^{2}}\right)^{2}
$$

\footnotetext{
${ }^{4}$ This can be easily seen by inspecting a player's reaction function. For the sake of simplicity consider the Cournot game when $N=2$. In this case, player $i$ 's reaction function is given by $q_{i}\left(q_{j}, r_{i}\right)=\left(a-\theta q_{j}\right) /\left(2-c+\theta r_{i}\right)$. Thus, the lower is a player's conjecture $r_{i}$, the higher the output in the Cournot game.
} 
To compute the equilibrium prices, we have to solve the system of first order conditions given conjectures. The solution of this system of equations yields $p_{1}^{*}\left(r_{1}, r_{2}\right)$ and $p_{2}^{*}\left(r_{1}, r_{2}\right)$, that is, the optimal prices given the conjectures of the firms. These equilibrium prices are quite complex so we refrain from writing them down. The profits implied by $p_{1}^{*}\left(r_{1}, r_{2}\right)$ and $p_{2}^{*}\left(r_{1}, r_{2}\right)$ are

$$
\begin{aligned}
& \pi\left(p_{1}^{*}\left(r_{1}, r_{2}\right), p_{2}^{*}\left(r_{1}, r_{2}\right)\right) \\
& \quad=p_{1}^{*}\left(\frac{a(1-\theta)-p_{1}^{*}+\theta p_{2}^{*}}{1-\theta^{2}}\right)-\frac{c}{2}\left(\frac{a(1-\theta)-p_{1}^{*}+\theta p_{2}^{*}}{1-\theta^{2}}\right)^{2}
\end{aligned}
$$

A conjecture $r^{*}$ is an ESS for $N=2$ if it solves the following maximization problem

$$
\max _{r}\left(\pi^{*}\left(r, r^{*}\right)-\pi^{*}\left(r^{*}, r\right)\right) .
$$

Assuming a symmetric equilibrium, i.e. $r=r^{*}$, the first-order condition is given by

$$
\frac{\left(r^{*}+1\right)\left(r^{*} \theta(c-\theta+1)-2+\theta(\theta+1)-c\right)(\theta+1)(\theta-1)^{2} \theta^{2} a^{2}}{\left(2 \theta r^{*}(c+2)\left(c-\theta^{2}+1\right)+\left(r^{*}\right)^{2} \theta^{2}\left(2 c+c^{2}+\theta^{2}+1\right)-\left(c-3 \theta+\theta^{2}+2\right)\left(c+3 \theta+\theta^{2}+2\right)\right)^{2}}=0 .
$$

Hence, the two candidate solutions are given by by $r_{1}^{*}=-1$ and $r_{2}^{*}=$ $\frac{c-\theta(\theta+1)+2}{\theta(c-\theta+1)}$.

To check the second-order condition, note that since $0<\theta<1$

$$
\begin{aligned}
& \left.\frac{\partial^{2}\left(\pi\left(r, r^{*}\right)-\pi\left(r^{*}, r\right)\right)}{\partial r^{2}}\right|_{r=r^{*}=-1} \\
& =-\frac{\theta^{2} a^{2}(c+2(1-\theta))(\theta+1)^{2}(\theta-1)^{2}}{\left(6 \theta(\theta-c)+4 \theta\left(\theta^{2}-1\right)+\theta c\left(c \theta+2\left(\theta^{2}-c\right)\right)-(c+2)^{2}\right)^{2}}<0 .
\end{aligned}
$$

Furthermore,

$$
\operatorname{sign}\left(\left.\frac{\partial^{2}\left(\pi\left(r, r^{*}\right)-\pi\left(r^{*}, r\right)\right)}{\partial r^{2}}\right|_{r=r^{*}=\frac{c-\theta(\theta+1)+2}{\theta(c-\theta+1)}}\right)=-\operatorname{sign}(2(\theta-1)-c)>0 .
$$

\section{References}

Bresnahan TF (1981) Duopoly models with consistent conjectures. Am Econ Rev 71:934-945

Dixon HD, Somma E (2003) The evolution of consistent conjectures. J Econ Behav Organ 51:523-536

Güth W, Huck S (1997) A new justification of monopolistic competition. Econ Lett 57:177-182

Hehenkamp B, Leininger W, Possajennikov A (2004) Evolutionary equilibrium in tullock contests: spite and overdissipation. Eur J Polit Econ 20:1045-1057

Maynard-Smith J (1982) Evolution and the theory of games. Cambridge University Press, Cambridge, UK

Müller W, Normann H-T (2005) Conjectural variations and evolutionary stability: a rationale for consistency. JITE, J Inst Theor Econ 161:491-502

Possajennikov A (2000) On the evolutionary stability of altruistic and spiteful preferences. J Econ Behav Organ 42:125-129

Springer 
Possajennikov A (2004) Evolutionary stability of constant consistent conjectures. Discussion Paper 04/03, University of Nottingham, Nottingham, UK

Schaffer ME (1988) Evolutionary stable strategies for a finite population and variable contest size. J Theor Biol 132:469-478

Vega-Redondo F (1997) The evolution of Walrasian Behavior. Econometrica 65:375-384 